\title{
TRATAMENTO DE HEMANGIOMA EM MUCOSA LABIAL POR ESCLEROTERAPIA: RELATO DE CASO CLÍNICO
}

\author{
Treatment of hemangioma in labial mucosa by sclerotherapy: \\ clinical case report
}

\author{
Emerson Lucas da Silva Coimbraa, (D) Mariana Pedreira Panceirob, (D) Felipe Lage da Rochac, \\ Roberto Gomes dos Santos d, (D) Gustavo Gaffrée Braz ${ }^{c}$, (D) Monica Lage da Rocha ${ }^{e}$
}

\section{RESUMO}

O hemangioma é uma lesão vascular, podendo representar-se como uma neoplasia benigna dos vasos sanguíneos, relativamente rara na cavidade oral, podendo causar prejuízo estético e funcional a depender da sua região. Sua localização mais frequente é o lábio superior, mas pode ocorrer em outras regiões, como língua, mucosa jugal e palato. O seu tratamento depende, principalmente, do correto diagnóstico da lesão, bem como da localização anatômica da mesma e pode ser realizado com esclerose química, crioterapia, laserterapia, excisão cirúrgica convencional, embolia arterial ou eletrocautério. O objetivo desse trabalho é apresentar um caso clínico, de um paciente do gênero masculino, 63 anos de idade, apresentando hemangioma desde os 16 anos de idade, com recidiva há dez anos em lábio superior. Ao exame físico intrabucal observou-se uma lesão tumoral, de coloração arroxeada, base séssil, assintomática, com limites bem definidos, medindo aproximadamente $4 \mathrm{~cm}$ em seu maior diâmetro, localizada na mucosa labial superior. Foi realizada manobra de vitropressão que apresentou um esmaecimento da coloração arroxeada, permitindo diagnóstico clínico de hemangioma. O tratamento proposto foi a esclerose terapêutica com cinco aplicações de oleato de monoetanolamina 5\%, considerando as características clínicas e os métodos de diagnóstico desta lesão. Por meio de um diagnóstico preciso e uma conduta terapêutica adequada, o caso apresenta-se com um acompanhamento de dois meses com significativa redução até o momento, e com um resultado estético funcional satisfatório. Conclui-se que a eficácia da escleroterapia realizada com o uso do oleato de monoetanolamina 5\% é uma alternativa terapêutica segura.

Palavras-chaves: Hemangioma. Medicina Bucal. Escleroterapia.

\begin{abstract}
Hemangioma is a vascular lesion, and may represent a benign neoplasm of blood vessels, relatively rare in the oral cavity, and may cause aesthetic and functional impairment depending on its region. Its most frequent location is the upper lip, but may occur in other regions, such as tongue, jugal mucosa and palate. Its treatment depends mainly on the correct diagnosis of the lesion, as well as its anatomical location and can be performed with chemical sclerosis, cryotherapy, laser therapy, conventional surgical excision, arterial embolism or electrocautery. The aim of this paper is to present a case report of a 63-year-old male patient with hemangioma since he was 16 years old, with a recurrence of the upper lip for 10 years. Physical examination revealed an asymptomatic purplish-colored, lesion-shaped tumor with well-defined limits, measuring approximately $4 \mathrm{~cm}$ in its largest diameter, located in the upper lip mucosa. A vitropression maneuver was performed that showed a faint purplish coloration, allowing a clinical diagnosis of hemangioma. The proposed treatment was therapeutic sclerosis with five applications of 5\% monoethanolamine oleate, considering the clinical characteristics and diagnostic methods of this lesion. Through an accurate diagnosis and an appropriate therapeutic approach, the case presents a two-month follow-up with significant reduction so far, and a satisfactory functional aesthetic result. It is concluded that the effectiveness of sclerotherapy performed with the use of $5 \%$ monoethanolamine oleate is a safe therapeutic alternative.
\end{abstract}

Keywords: Hemangioma. Oral medicine. Sclerotherapy.

\footnotetext{
${ }^{a}$ Graduado em Odontologia, Universidade Salgado de Oliveira, RJ, Brasil.

${ }^{\mathrm{b}}$ Graduada em Odontologia, Universidade Salgado de Oliveira, RJ, Brasil.

${ }^{\mathrm{c}}$ Especialista em Cirurgia Bucomaxilofacial, Hospital Municipal Lourenço Jorge, RJ, Brasil.

${ }^{\mathrm{d}}$ Mestre em DTM e Dor Orofacial, São Leopoldo Mandic, SP, Brasil.

${ }^{e}$ Doutora em Patologia, Universidade Federal Fluminense, RJ, Brasil.

Autor de correspondência: Emerson Lucas da Silva Coimbra - E-mail: dremersoncoimbra@gmail.com

Data de envio: 01/10/2019 | Data de aceite: 16/12/2019
} 


\section{INTRODUÇÃO}

O hemangioma é uma neoplasia vascular benigna, caracterizado por uma fase de crescimento rápido, com proliferação de células endoteliais, seguida pela involução gradual ${ }^{1-3}$. A localização mais comum é a região da cabeça e do pescoço, ocorrendo em $60 \%$ dos $\operatorname{casos}^{1,2}$.

Essa lesão normalmente é encontrada na infância, de forma congênita, embora possa desenvolver-se em adultos, tem predileção pelo gênero feminino e ocorre com maior frequência em indivíduos leucodermas ${ }^{2,4}$. Sua etiologia ainda é incerta, sendo que estímulos endócrinos e inflamatórios podem desencadear essa proliferação irregular, que em alguns casos pode ser também herdada 5 .

De uma forma geral, o hemangioma apresenta-se como lesão única, podendo ser observado em qualquer parte do organismo, sendo comum na região de cabeça e pescoço. No entanto, é relativamente raro na cavidade oral. Na cavidade oral, as localizações mais comuns são nos lábios, língua e mucosa jugal ${ }^{1,2,4,6,7}$. Clinicamente, o hemangioma pode apresentar-se de diversas formas, sendo observado, geralmente, em forma de bolha preenchida completamente por conteúdo sanguíneo, apresentando uma coloração azul-violácea que empalidece e diminui de tamanho à compressão $0^{1,4}$.

Essas lesões podem ser muitas vezes diagnosticadas clinicamente pelas suas características de cor, forma e patogenia, e por técnicas, como a compressão bidigital e vitropressão (diascopia), devido ao fato da biópsia excisional estar contraindicada pelo potencial de risco de hemorragias importantes, difíceis de coibir pelos métodos hemostáticos de rotina ${ }^{7-9}$.

A diascopia ou vitropressão constitui um importante auxiliar no estabelecimento do diagnóstico diferencial. À compressão feita pela lâmina de vidro, faz com que a lesão adquira coloração pálida momentaneamente, diminuindo de tamanho devido ao esvaziamento vascular e posteriormente voltando ao seu volume inicial após a remoção da lâmina, podendo-se assim, estabelecer o diagnóstico clínico sugestivo, eliminando hipóteses de quaisquer lesões pigmentadas ${ }^{1,10,11}$.

O hemangioma pode ser classificado histologicamente em capilar ou cavernoso e, apesar de existirem outras classes de hemangioma, estas serão variações dos dois tipos básicos ${ }^{1,72,1213}$.

O tratamento do hemangioma pode ser realizado por diferentes formas terapêuticas conforme a localização, extensão e etiologia da lesão. Na literatura observa-se, em casos congênitos, o uso da proservação devido à possibilidade de total remissão com a idade, contudo, se a involução espontânea não for uma opção, pode-se realizar infiltração de solução química esclerosante, crioterapia, laserterapia, corticoterapia e embolia vascular, cada uma isoladamente ou de forma combinada com a excisão cirúrgica ${ }^{11,12,14,15}$. Dentre as possíveis terapêuticas existentes, a esclerose por infiltração de agente químico é uma das modalidades terapêuticas mais utilizadas, uma vez que tem como vantagem ser um procedimento pouco invasivo e não promover cicatriz aparente. Apresenta poucas complicações, porém traz a desvantagem de não poder ser utilizada em lesões de grandes dimensões, pois o fluxo sanguíneo rápido e os espaços vasculares largos e difundidos acabam por diluir e proporcionar um pequeno contato com as paredes vasculares, ocasionando efeito mínimo ou ausente do agente químico com o endotélio vascular ${ }^{11,12,16}$ Entre as substâncias químicas possíveis de serem utilizadas para a infiltração, o oleato de monoetanolamina à $5 \%$ - ethamolin ${ }^{\circledR}$ apresenta-se como agente mais utilizado devido a sua menor toxicidade ${ }^{15}$. Na ausência de consequências estéticas ou funcionais, a proservação é a melhor conduta a ser adotada ${ }^{10}$.

O objetivo do presente trabalho é apresentar um caso clínico de hemangioma oral, onde o tratamento de escolha foi a esclerose com oleato de etanolamina 5\%, substância no qual é uma alternativa terapêutica minimamente invasiva. 


\section{RELATO DE CASO}

Paciente J.L.P do gênero masculino, 63 anos de idade, leucoderma. Procurou o serviço de Estomatologia do Hospital Rocha Maia no Rio de Janeiro, com queixa de aumento de volume no lábio superior que incomodava na estética. Durante a anamnese o paciente relatou que estas lesões estavam presentes desde os 16 anos de idade, no qual foi realizado escleroterapia, com recidiva há dez anos no mesmo local.

Ao exame clínico intrabucal observou-se a presença de uma lesão nodular, arroxeada, de base séssil, superfície lisa, consistência macia, assintomática, não pulsátil, com limites bem definidos, na mucosa labial superior do lado esquerdo estendendo-se para a mucosa labial interna, medindo aproximadamente $4 \mathrm{~cm}$ em seu maior diâmetro, sem presença de úlcera traumática (Figura 1).

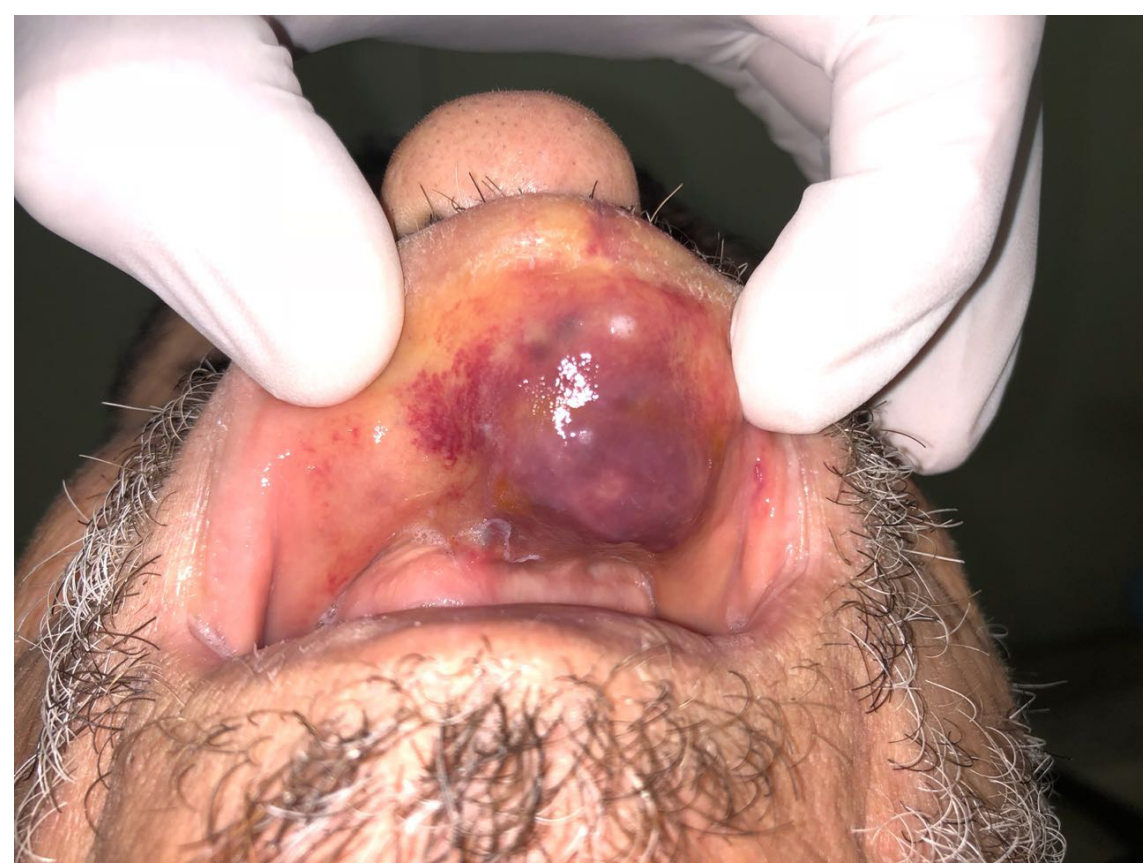

Figura 1: Aspecto clínico inicial da lesão nodular em mucosa labial superior.

Devido às características clínica da lesão encontrada, chegou-se à hipótese diagnóstica de lesão vascular. Dessa forma foi aplicado o teste de vitropressão, no qual realizou-se uma compressão com uma lâmina de vidro. Com essa manobra, a mancha adquiriu uma coloração pálida, diminuindo de tamanho devido ao esvaziamento vascular. Ficou definido, assim, o diagnóstico de hemangioma.

Devido a grande extensão da lesão, o plano de tratamento estabelecido foi a realização da esclerose química, utilizando $1 \mathrm{ml}$ de oleato de monoetanolamina (Ethamolin ${ }^{\circledR}$ ) 0,05g/ml, diluído em cloridrato de lidocaína a $2 \%$ com vasoconstritor, aplicado intralesionalmente até o desaparecimento das lesões ou até obter um efeito cosmético satisfatório. Foram realizadas 4 aplicações, com intervalos de 15 dias. As aplicações foram realizadas com seringas de $1 \mathrm{ml}$ e agulha de insulina, através de uma injeção lenta e gradual.

A primeira aplicação foi de $1 \mathrm{ml}$ de oleato de monoetanolamina na porção mais profunda da lesão. Quinze dias após observou-se uma diminuição do tamanho das lesões em 30\%, bem como da coloração arroxeada, sem presença de úlcera traumática. Durante a aplicação, não foi observada qualquer sensação incômoda para o paciente. Na consulta pós-terapêutica 
(após 7 dias), não foi observada nenhuma úlcera traumática na região de mucosa labial, sendo a regressão da lesão e a diminuição da coloração arroxeada bem evidente. Na terceira aplicação já podia observar uma redução da lesão em 50\% e ausência de dor (Figura 2). Na quarta e última aplicação foi observado uma redução em 95\% da lesão. 15 dias após a última aplicação, observou-se regressão total da lesão, com presença de fibrose residual e ausência cicatrizes pós-procedimento, obtendo um resultado estético favorável (Figura 3).

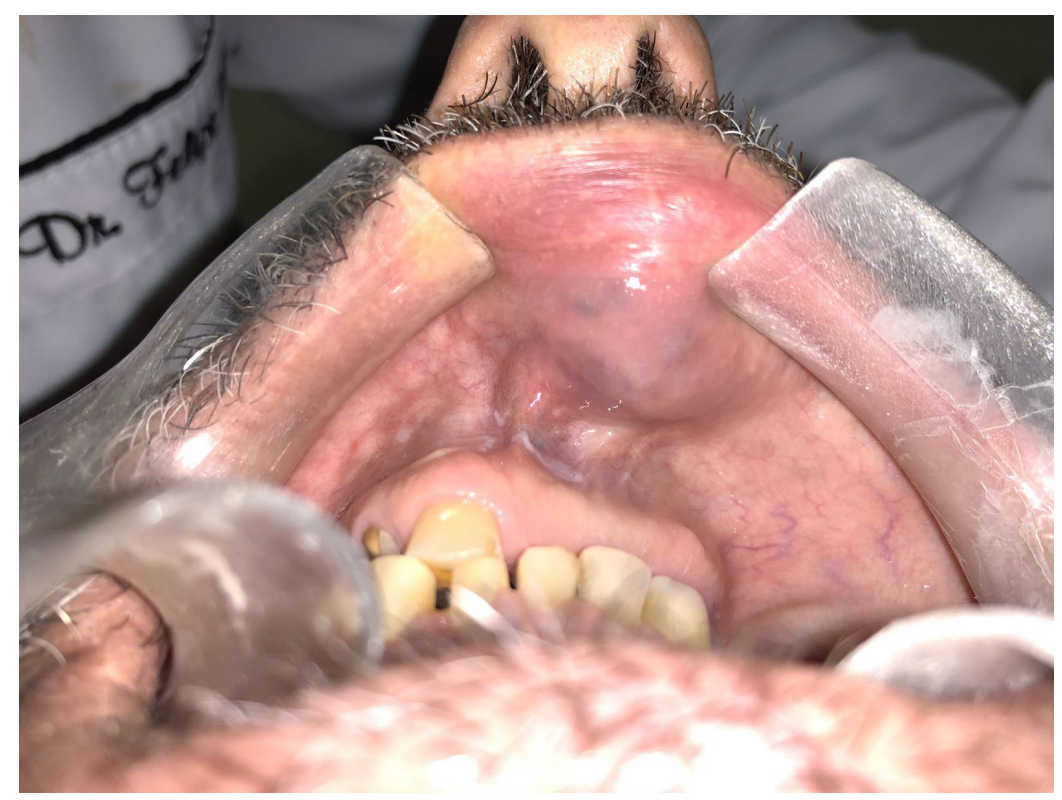

Figura 2: Aspecto clínico da lesão após a terceira sessão, com redução de 50\% da lesão.

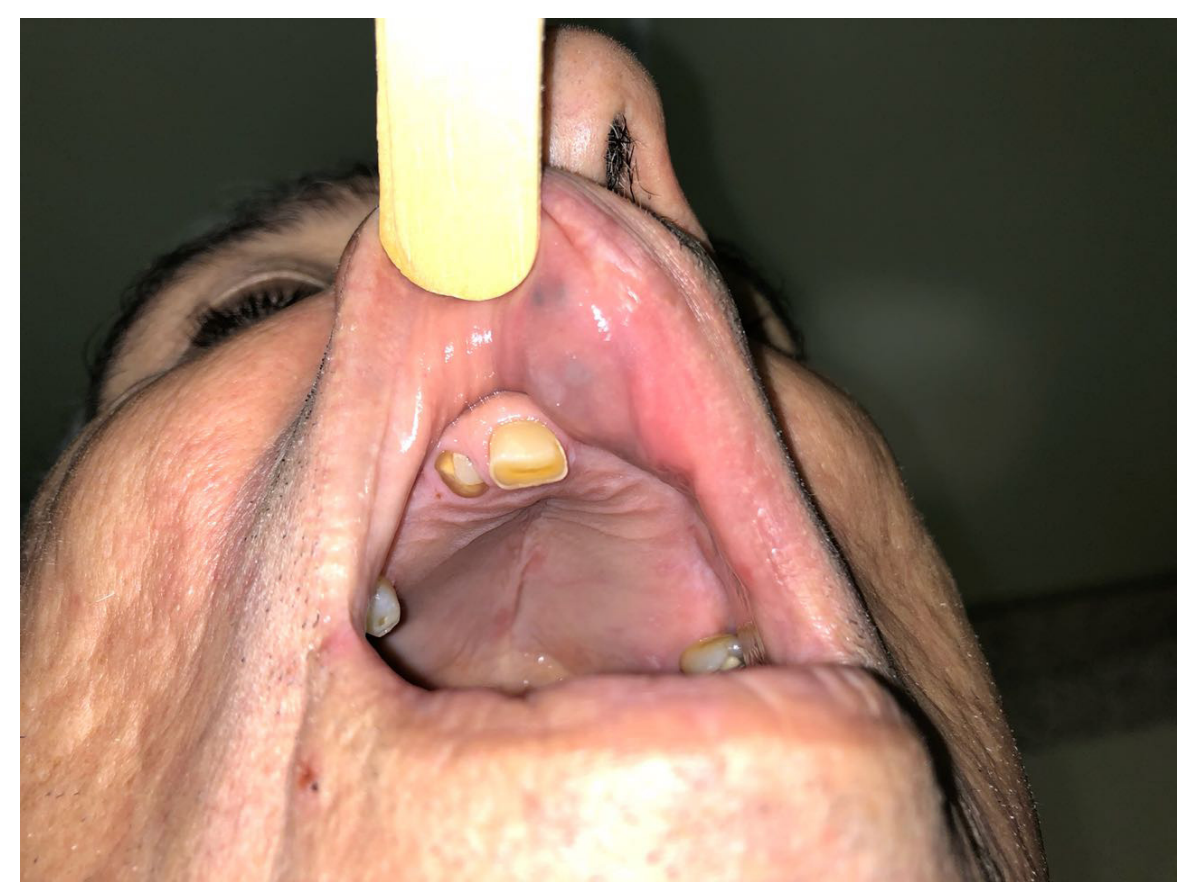

Figura 3: Aspecto da lesão onde é observado regressão total, com presença de fibrose residual e ausência cicatrizes pós-procedimento. 
Foi sugerido um acompanhamento semestral para avaliar a possibilidade de outra aplicação do oleato de etanolamina. O paciente encontra-se proservado por um período de 6 meses, e, até o presente momento, não se constatou recidiva das lesões.

\section{DIscussão}

O hemangioma faz parte do grupo das alterações vasculares benignas. É mais comum na região de cabeça e pescoço e pode ser encontrado na cavidade oral causando ulceração, dor, sangramento, infecção secundária e deformação do tecido ${ }^{17-19}$. É mais comum em crianças e poucos casos dá-se em adultos. Mulheres são mais afetadas. Lábios, língua, mucosa jugal e palato são os locais mais frequentemente envolvidos ${ }^{17-19}$.

A etiologia dos hemangiomas está ligada a anomalias congênitas, traumas físicos, estímulos endócrinos e inflamatórios de etiologia desconhecida. O prognóstico da hemangioma, em geral, é excelente, uma vez que não tende a reaparecer ou sofrer transformação maligna, após o tratamento adequado ${ }^{20}$.

Clinicamente apresentam-se como uma mancha ou nódulo arroxeado, cuja coloração varia de vermelho intenso ao roxo, de acordo com a localização, a profundidade no tecido e o grau de congestão da área afetada. Em geral, essas lesões são relativamente flácidas à palpação, podendo ser difusa ou circunscrita, apresentando-se plana ou elevada, com superfície nodular ou lisa e assintomática na maioria das vezes. O seu tamanho é variável, dependendo da idade do paciente e da localização da lesão, pois a fase proliferativa do tumor frequentemente dura de 6 a 10 meses. Depois, ele cresce lentamente e tende a involuir para o tamanho inicial ${ }^{1,21-23}$. Ainda que raro, o hemangioma também podem ser intra-ósseo, com características radiográficas de lesão radiolúcida multilocular com aspecto de "favos de mel”7,23. O caso clínico relatado compartilha algumas destas características clínicas descritas, como por exemplo: localização, aspeto nodular e coloração arroxeada.

Uma das complicações mais comuns nos casos de hemangiomas é a ulceração, a qual pode ocorrer com ou sem infecção secundária³. A principal queixa dos pacientes portadores de hemangiomas é o distúrbio estético que, dependendo do tamanho e da localização, podem ocasionar assimetria facial ou interferir na função dos órgãos envolvidos ${ }^{24-26}$.

O diagnóstico do hemangioma, exceto nos casos intraósseos, pode ser estabelecido de forma simples e segura pela anamnese, exame clínico e por manobras semiotécnicas, como a vitropressão, que são conclusivas na maioria dos casos ${ }^{7}$. Por se tratar de uma lesão vascular, a realização de biópsia incisional está contraindicada nestes casos devido ao risco de hemorragias ${ }^{27,28}$. A vitropressão é importante exame auxiliar no estabelecimento do diagnóstico diferencial. À compressão pela lâmina de vidro, o hemangioma adquire coloração pálida, diminuindo de tamanho devido ao esvaziamento vascular ${ }^{7}$. No caso clínico apresentado, realizamos a diascopia confirmando uma lesão vascular, ressaltando que o diagnóstico definitivo de hemangioma, somente poderia ser feito através da biópsia.

Para a escolha do tipo de tratamento dos hemangiomas algumas características devem ser levadas em consideração como tamanho, localização e duração da lesão, idade do paciente, hemodinâmica da lesão, através da observação do fluxo sanguíneo, além da viabilidade da técnica a ser utilizada ${ }^{8,28,29}$. Verificamos, entre os autores consultados, várias opções terapêuticas, entre elas: a excisão cirúrgica convencional, eletrocauterização, laserterapia, embolização, crioterapia e escleroterapia química ${ }^{3}$.

De acordo com os autores consultados na revisão da literatura, a escleroterapia é uma técnica efetiva, não invasiva e conservadora para o tratamento de lesões vasculares benignas ${ }^{8,17}$, tendo sido adotada para o caso clínico relatado, uma vez que a lesão era de tamanho 
médio, e de fácil acesso para a aplicação e mais confortável para a paciente. O agente esclerosante é um derivado do ácido oleico, com propriedades hemostáticas ${ }^{8,17}$. O componente oleico provoca a coagulação local por meio da ativação do fator de Hagemman e a etanolamina inibe a formação do coágulo de fibrina pela quelação do cálcio e a ação conjunta das 2 substâncias permite um equilíbrio hemostático, evitando a hemorragia após a administração nas lesões vasculares ${ }^{8,17}$. Portanto, seu mecanismo de ação baseia-se na produção de uma irritação, uma resposta inflamatória e fibrose endotelial e possível oclusão da veia ${ }^{8,17}$. As concentrações do oleato de etanolamina devem ser cuidadosamente observadas, pois $5 \%$ da substância pode causar toxidade renal associada a hemólise vascular e hemoglobinúria ${ }^{30}$. Para evitar esta possível complicação renal sugere-se aplicações com menos de $1 \mathrm{ml}$ de $5 \%$ do oleato de etanolamina ${ }^{17}$.

A quantidade e o intervalo da administração do oleato de monoetanolamina a 5\% devem ser proporcionais ao tamanho da lesão, preconizando-se, de modo geral, que sua aplicação seja realizada em sessões intercaladas com no mínimo sete dias entre cada aplicação, não ultrapassando $2 \mathrm{ml}$ por infiltração ${ }^{8,17}$. Está contra indicado em casos de pacientes diabéticos não controlados e em áreas com infecção secundária. A utilização do oleato de etanolamina deve ser evitada durante a gravidez, pois pode ter efeito iatrogênico, não tendo ainda sido estabelecida sua segurança ${ }^{30-32}$.

No presente trabalho, optou-se pela escleroterapia química com oleato de etanolamina, por ser um método seguro, com a possibilidade de regressão total ou parcial da lesão, e por excelentes resultados encontrados na literatura.

\section{CONCLUSÃo}

De acordo com a literatura e resultados obtidos conclui-se que: escleroterapia com oleato de etanolamina 5\% é uma alternativa segura, de baixo custo, eficaz e de menor morbidade para pacientes com hemangiomas orais. O caso apresentado apresenta várias características clássicas desta neoplasia benigna, como a recidiva após o tratamento, a localização e o aspecto clínico e a excelente resposta à terapêutica esclerosante. Com relação ao sexo do paciente, porém, o caso difere da literatura, que apresenta maior prevalência no sexo feminino. A partir das características apresentadas no caso relatado, pode-se notar a importância da vitropressão no diagnóstico diferencial das lesões de origem vascular.

\section{REFERÊNCIAS}

1. Neville BW, Damm DD, Allen CM, Bouquot JE. Patologia oral e maxilofacial. 4. ed. Rio de Janeiro: Guanabara -Koogan; 2016.

2. El-Naggar AK, Chan JKC, Grandis JR, Takata T, Slootweg P], editors. Geneva: World Health Organization; 2017. [acesso 2019 Set 25]. Disponível em: http://publications.iarc.fr/Book-And-Report-Series/Who-larc-Classification -Of-Tumours/Who-Classification-Of-Head-And-Neck-Tumours-2017.

3. Bonet-Coloma C, Minguez-Martínez I, Palma-Garrió C, Galán-Gil S, Peñarrocha- Diago M, Minguez-Sanz JM. Clinical characteristics, treatment and outcome of 28 oral haemangiomas in pediatric palients. Med Oral Patol Oral Cir Bucal. 2011;16(1):19-22.

4. Assis GM, Silva SRP, Moraes PH, Amaral JIQ, Germano AR. Hemangioma de língua: relato de caso. Rev Cir Traumatol Buco-Maxilo-fac. 2009;9(2):59-66. 
5. Cruz FLG, Carvalho RF, Carvalho MF, Sales LAR, Devito KL. Diagnóstico diferencial de hemangioma por meio da vitropressão. Rev Gaúcha Odontol. 2011;59(1):125-9.

6. Johann AC, Aguiar MC, do Carmo MA, Gómez RS, Castro WH, Mesquita RA. Sclerotherapy of benign oral vascular lesion with ethanolamine oleate: an open clinical trial with 30 lesions. Oral Surg Oral Med Oral Pathol Oral Radiol Endod. 2005;100(5):579-84.

7. Barret AW, Speight PM. Superficial arteriovenous hemangioma of the oral cavity. Oral Surg, Oral Med, Oral Pathol, Oral Radiol, Endod 2000;90:731-8.

8. Gontijo B, Silva CMR, Pereira LB. Hemangioma da infância. Anais Brasileiros de Dermatologia 2003;78(6):651-73.

9. Rocha LB, Pádua ], Martins RH, Lia R. Hemangioma da cavidade bucal. RGO Rev gauch odontol. 2000;48(3):150-2.

10. Selim H, Selim A, Kachemoune A, Metwally SAFA. Use of esclerosing agent in the management of oral and peri-oral hemangiomas: review and case reports. Med Sci Monit 2007;13(9):114-9.

11. Chan C, Iv M, Fischbein N, Dahmoush H. Lobular capillary hemangioma of the mandible: a case report. Clinical Imaging. 2018;50:246-9.

12. Seo], Utumi E, Zambon C, Pedron I, Rocha A. Escleroterapia de hemangioma labial. Rev Odonto 2009;17(34):106-8.

13. Rane S, Parkhi MV, Agrawal G, Ghodke A. Cavernous hemangioma of uvula: a rare site. Indian ] Pathol Microbiol 2019;62:631-2.

14. Boraks S. Medicina bucal tratamento clínico-cirúrgico das doenças bucomaxilofaciais. 1. ed. São Paulo: Artes Médicas; 2011.

15. Hassani A, Khojasteh A, Vahid R. Management of mandibular vascular malformation with sclerotherapy. Report of two cases. Oral Surg Oral Med Oral Pathol Oral Radiol Endod 2006; 102(1):99-103.

16. Das BK, Hoque S. Treatment of venous malformations with ethanolamine oleate. Asian ] Surg 2008;31(4):22-4.

17. Choi $\mathrm{YH}$, Han $\mathrm{MH}, \mathrm{O}-\mathrm{Ki} \mathrm{K}$, Cha SH, Chang KH. Craniofacial cavernous venous malformations: percutaneous sclerotherapy with use of ethanolamine oleate. J Vasc Interv Radiol 2002;13(5):475-82.

18. Johann ACBR, Aguiar MCF, Carmo AV, Gomez RS, Castro WH, Mesquita RA. Sclerotherapy of benign oral vascular lesion with ethanolamine oleate: an open clinical trial with 30 lesions. Oral Surg Oral Med Oral Pathol Oral Radiol Endod. 2005;100:579-84.

19. Gomes CC, Gomez RS, do Carmo MAV, Castro WH, Gala-Garcia A, Mesquita RA. Mucosal varicosities: case report treated with monoethanolamine oleate. Med Oral Pathol Cir Buccal. 2006;11:44-6.

20. Cappabianca S, del Vecchio W, Giudice A, Colella G. Vascular malformations of the tongue: MRI findings on three cases. Dentomaxillofac Radiol. 2006;35:205-8.

21. Bharti V, Singh ]. Capillary hemangioma of palatal mucosa. ] Indian Soc Periodontol. 2012;16(3):475-8.

22. Assis GM, Silva SRP, Moraes PH, Amaral JIQ, Germano AR. Hemangioma de língua: relato de caso. Rev Cir Traumatol Buco-Maxilo-fac. 2009;9(2):59-66.

23. Bharti V, Singh ]. Capillary hemangioma of palatal mucosa. ] Indian Soc Periodontol. 2012;16(3):475-8.

24. Toledo HJB, Castro EVFL, Castro AL, Soubhia AMP, Salvador Júnior FB. Hemangioma cavernoso de lábio inferior: caso clínico. Rev Odontol araçatuba. 2004;25(1):9-11.

25. Seo], Utumi ER, Zambon CE, Pedron IG, Rocha AC. Escleroterapia de hemangioma labial. Odonto. 2009;17(34):106-12.

26. Tavares GR, Cavalcanti MOA, Tavares SSS, Aragão MS. Hemangiomas múltiplos na boca. Rev Bras Ci Saúde. 2009;13(1):77-81.

27. Cardoso LC, Fernandes LMPSR, Rocha JF, Gonçales ES, Ferreira Júnior O, Taveira LaS. Abordagem cirúrgica de hemangioma intraoral. Odontol Clín-Cient. 2010;9(2):177-80.

28. Loureiro CC, Falchet PC, Gavranich ] Jr, Lobo Leandro LF. Embolization as the treatment for a life-threatening mandibulararteriovenous malformation. J Craniofac Surg. 2010;21(2):380-2.

29. Wang M, Chen S, Mojica WD, Chen F. Cavernous hemangioma of the uvula: report a rare case with literature review. N A] Med Sci 2015;8:56-8.

30. Akita S, Akino K, Tanaka K, Anraku K, Yano H, Hirano A. Therapeutic choice for craniofacial venous malformations. ] Craniofac Surg. 2006;17:729-35.

31. Queiroz SIML, Assis GM, Silvestre VD, Germano AR, Silva JSP. Treatment of oral hemangioma with sclerotherapy: case report. J vasc Bras. 2014;13(3):249-53.

32. Pereira CCT, Figueiredo MAZ, Cherubini K. Tratamento de hemangioma bucal com agente esclerosante. JBC. 2002;6:324-2. 